\title{
Maternal smoking during pregnancy and daughters' risk of gestational diabetes and obesity
}

\author{
K. Mattsson • K. Källén • M. P. Longnecker • A. Rignell-Hydbom • L. Rylander
}

Received: 19 February 2013 / Accepted: 26 April 2013 /Published online: 23 May 2013

(C) Springer-Verlag Berlin Heidelberg (outside the USA) 2013

\begin{abstract}
Aims/hypothesis The primary aim of the study was to investigate the risk of developing gestational diabetes in women who were exposed to tobacco smoke in utero. Secondary aims were to assess the risk of obesity and non-gestational diabetes.

Methods Data were retrieved from the Medical Birth Register of Sweden for women who were born in 1982 (when smoking data were first registered) or later and who had given birth to at least one child; 80,189 pregnancies were included. The associations between in utero smoking exposure (three categories: non-smokers, 1-9 cigarettes/day [moderately exposed] and $>9$ cigarettes/day [heavily exposed]) and subsequent gestational diabetes $(n=291)$, non-gestational diabetes $(n=280)$ and obesity $(n=7,300)$ were assessed.

Results The adjusted ORs (aORs) of gestational diabetes were increased among women who were moderately (1.62, $95 \%$ CI $1.24,2.13)$ and heavily $(1.52,95 \%$ CI $1.12,2.06)$ exposed. The corresponding aORs of obesity were 1.36 (95\% CI $1.28,1.44)$ and $1.58(95 \%$ CI $1.48,1.68)$, respectively. A reduced OR for non-gestational diabetes was seen in the offspring of heavy smokers (aOR $0.66,95 \%$ CI $0.45,0.96$ ). Conclusions/interpretation Women exposed to smoking during fetal life were at higher risk of developing gestational diabetes and obesity.
\end{abstract}

Electronic supplementary material The online version of this article (doi:10.1007/s00125-013-2936-7) contains peer-reviewed but unedited supplementary material, which is available to authorised users.

K. Mattsson $(\varangle) \cdot K$. Källén · A. Rignell-Hydbom • L. Rylander Division of Occupational and Environmental Medicine, Institute of Laboratory Medicine, Lund University, SE-221 85 Lund, Sweden e-mail: kristina.mattsson@med.lu.se

M. P. Longnecker

Epidemiology Branch, National Institute of Environmental Health Sciences, NIH, DHHS, Research Triangle Park, NC, USA
Keywords Diabetes mellitus · Gestational diabetes . Intrauterine exposure $\cdot$ Maternal smoking $\cdot$ Medical Birth Register · Obesity
Abbreviations
G1 Generation 1
G2 Generation 2
GDM Gestational diabetes
MBR Medical Birth Register

\section{Introduction}

The developmental origins of health and disease have lately been receiving growing research interest. Preventable environmental exposures, such as maternal smoking during pregnancy, are of particular importance in this context. Exposure to smoking in utero has repeatedly been shown to detrimentally influence the offspring, with short-term effects that include fetal growth restriction, shortened gestational length and an increased risk of perinatal mortality $[1,2]$. Studies also indicate more long-term consequences such as obesity in children [3-5]. While the relation of intrauterine tobacco exposure to outcomes in childhood has been much studied, reports on possible adverse effects that persist until adulthood are more scarce and results are inconsistent [6-8].

Norwegian women who reported that their mothers had been smoking while they were pregnant with them had a higher risk of developing gestational diabetes mellitus (GDM) compared with non-exposed women [6]. This is, to our knowledge, the only study of prenatal tobacco exposure and subsequent gestational diabetes. The cohort, however, included only $38.5 \%$ of all women invited to participate, and information on the participants' exposure to prenatal smoking was obtained by asking them if their mothers smoked while 
pregnant with them. Further, evaluation of a dose-response relationship was not possible with those data.

The Swedish Medical Birth Register (MBR) has been collecting information on smoking during pregnancy since 1982, and now some of the women born that year or later have become pregnant and given birth, yielding a large cohort suitable for examining the earlier results and elucidating details about the associations.

The primary objective of this study was to further examine these long-term effects by studying, using a populationbased national register, the risk of GDM development in women who were exposed to tobacco smoking in utero. As a second aim the risks of non-gestational diabetes and obesity were investigated. Data from experiments in animals [9] suggest that in utero exposure to tobacco smoke would be associated with an increased risk of GDM, but not type 1 diabetes mellitus, for which non-gestational diabetes was a proxy in the present study.

\section{Methods}

Data selection The data for this study were derived from the MBR of Sweden. The register has information on nearly all births in Sweden since 1973. Data collection begins with the woman's first antenatal visit in early pregnancy (usually at 8 12 weeks of gestation). The register has a high level of completion; records are missing for $0.5-3.9 \%$ of all births [10]. In 1982, the register began recording information on smoking, and thus the cohort used here consists of women who were born in 1982 or later, who have given birth to at least one child of their own. Data were retrieved through 2010. Of a total of 100,175 eligible pregnancies, those with missing data on smoking behaviour in either generation 1 (G1) or generation 2 (G2) were excluded, leaving a final sample of 80,189 pregnancies. Most of the exclusions were due to missing smoking data for G1. Some of the women contributed with more than one pregnancy $(27.3 \%)$. The study was approved by the ethics board at Lund University, Sweden.

Exposure assessment Women (both G1 and G2) were interviewed by trained midwives using a standardised questionnaire; they reported their current smoking behaviour during their first antenatal visit (in Sweden there are typically two visits per woman if the pregnancy is without complications). They are categorised in the MBR as nonsmoker, 1-9 cigarettes/day (G2 offspring hence referred to as moderately exposed) or $>9$ cigarettes/day (G2 offspring hence referred to as heavily exposed). There are data in the MBR on smoking status 3 months before pregnancy, based on maternal self-reporting at the first antenatal visit, as well as information on current maternal smoking during the later part of pregnancy collected at the second antenatal visit (at
30-32 weeks of gestation). However, these variables have been added to the register later (data available from 1999 for pre-pregnancy smoking and from 1990 for smoking in the last trimester). Thus, few individuals had valid data on these variables (72.5\% missing during the years 1990-1999 for last-trimester smoking) and therefore those variables were not used in the present study. There was no possibility of examining if the timing of the first antenatal visit influenced smoking status because it is not recorded in the MBR at what week of gestation the antenatal visit occurs.

Outcomes The main outcomes (in G2 women) were GDM, non-gestational diabetes and obesity. BMI was calculated from measured height and early pregnancy weight, recorded by the MBR. Obesity was defined here as a BMI $\geq 30 \mathrm{~kg} / \mathrm{m}^{2}$. MBR registers pregnancy-related diagnoses using the ICD; during the time frame studied, ICD versions 8,9 (www.icd9data.com/2007/Volume1/240-279/250-259/250/ default.htm) and 10 (www.who.int/classifications/icd/en/) were used. Using the ICD codes, we classified women with any type of diabetes at the first antenatal visit as having nongestational diabetes; more precise categorisation as to type was not possible. Pregnant women without non-gestational diabetes and subsequently diagnosed with gestational diabetes were classified as having GDM.

GDM was registered in the MBR beginning in 1987. GDM is diagnosed in Sweden by a fasting $75 \mathrm{~g}$ OGTT, where fasting plasma glucose levels of $>5.5 \mathrm{mmol} / \mathrm{l}$ and $2 \mathrm{~h}$ plasma glucose levels of $>9.0 \mathrm{mmol} / \mathrm{l}$ constitute gestational diabetes. However, regional variation exists in terms of who is offered an OGTT; some regions administer the test to all pregnant women in the later part of pregnancy, whereas other regions do so only for women with risk factors for GDM [11].

Covariates Other covariates used in the analysis were: (G2) woman's age at pregnancy (three categories: $<20,20-24$, 25-28); parity (three categories: 1, 2 and $\geq 3$ ); own smoking in early pregnancy (three categories: non-smokers, 1-9 cigarettes/day and $>9$ cigarettes/day); BMI (six categories: $<18,18-24,25-29,30-34,35-39$ and $>40$ ); birthweight (eight categories: 1,500-1,999, 2,000-2,499, 2,500-2,999, 3,000-3,499, 3,500-3,999, 4,000-4,499, 4,500-4,999 and $5,000-5,500 \mathrm{~g}$ ); gestational age at delivery (four categories: $<32,32-36,37-42$ and $>42$ completed weeks of gestation); and mode of delivery (four categories: vaginal birth, elective Caesarean section, emergency Caesarean section and forceps/vacuum extraction). All of these variables were obtained from the MBR, and, for the variables own smoking and mode of delivery, the categories precoded by the MBR were used.

Statistical analysis Only G2 women with a BMI of 15-50 and birthweights of 1,500-5,500 $\mathrm{g}$ were included in the 
analyses. The associations between fetal tobacco exposure in early pregnancy and subsequent GDM, non-gestational diabetes and obesity in G2 women were assessed through separate logistic regressions generating ORs with $95 \%$ CIs.

The models were adjusted for the woman's (G2) age at pregnancy, parity, mode of delivery and own smoking in early pregnancy. As a second step, the models of GDM and non-gestational diabetes were also adjusted for G2 BMI. As birthweight and gestational age might be intermediate variables, through which intrauterine smoking exposure influences the outcomes, analyses that further adjusted for those two covariates were conducted for all models.

Because the results of a previous study indicated a possible interaction between fetal tobacco exposure during pregnancy (G1 smoking) and woman's own smoking in early pregnancy (G2 smoking) with respect to risk of obesity and gestational diabetes [6], we also evaluated this. An interaction was considered important if the $p$ value for the interaction term was below 0.10 . If this was the case, separate analyses were performed among G2 women who smoked in early pregnancy and among G2 women who did not, when the number of exposed cases was sufficient to allow the stratified analysis (a minimum of $n=5$ in each strata was considered a requirement).

To reduce a possible impact of heredity, we conducted separate analyses for non-gestational diabetes where we excluded the $\mathrm{G} 2$ women whose mothers (G1) had non-gestational diabetes. Corresponding analyses were performed for obesity. However, as information on GDM was only available since 1987, it was not possible to conduct the corresponding analysis for GDM.

Further, the following sensitivity analyses to evaluate the robustness of the results were performed: (1) including only G2 with a parity of 1 ; (2) including only women born in Sweden (G1); (3) excluding G2 women whose mothers had non-gestational diabetes from the GDM model; (4) additionally adjusting the models for G1 BMI. Further, as screening practices for GDM differ regionally in Sweden, another set of analyses were performed in which only G2 women delivering in the region of Scania were included (in this region all pregnant women receive an OGTT). All analyses were carried out using SPSS version 19.0 (SPSS, Chicago, IL, USA).

\section{Results}

The age range among the G2 women was 13-28 years, reflecting the requirement that they were born in 1982 or later and pregnant by 2010 (Table 1). The G2 generation was, on average, younger, more overweight and more likely to be primiparous than G1. The prevalence of GDM and non-gestational diabetes were roughly similar across the two
Table 1 Characteristics of included sample of women with complete smoking data in the two generations (total $n=54,012$ )

Smoking was recorded in the MBR beginning in 1982. Values are percentages

${ }^{\mathrm{a}}$ No cases in dataset

${ }^{\mathrm{b}}$ Gestational diabetes recorded since 1987.

For G1, the prevalence of GDM is calculated after that year

${ }^{\mathrm{c}}$ MBR does not distinguish between type 1 and type 2 diabetes

\begin{tabular}{|c|c|c|}
\hline Characteristic & G1 & $\mathrm{G} 2$ \\
\hline \multicolumn{3}{|c|}{ Age at childbirth (years) } \\
\hline$<20$ & 7.5 & 10.2 \\
\hline $20-24$ & 36.5 & 59.2 \\
\hline $25-29$ & 33.2 & 30.6 \\
\hline $30-35$ & 16.1 & $-^{\mathrm{a}}$ \\
\hline $35-40$ & 5.8 & - \\
\hline$>40$ & 1.0 & - \\
\hline \multicolumn{3}{|l|}{ BMI $\left(\mathrm{kg} / \mathrm{m}^{2}\right)$} \\
\hline$<18$ & 5.8 & 2.2 \\
\hline $18-24$ & 76.8 & 60.7 \\
\hline $25-29$ & 14.3 & 23.6 \\
\hline $30-34$ & 2.8 & 9.4 \\
\hline $35-39$ & 0.3 & 3.0 \\
\hline$>40$ & - & 1.1 \\
\hline \multicolumn{3}{|l|}{ Parity } \\
\hline 1 & 41.1 & 72.0 \\
\hline 2 & 32.9 & 24.2 \\
\hline 3 or higher & 26.0 & 3.8 \\
\hline \multicolumn{3}{|c|}{ Country of birth } \\
\hline Sweden & 90.5 & 100.0 \\
\hline Other & 9.5 & - \\
\hline \multicolumn{3}{|l|}{$\mathrm{GDM}^{\mathrm{b}}$} \\
\hline No & 99.6 & 99.5 \\
\hline Yes & 0.4 & 0.5 \\
\hline \multicolumn{3}{|c|}{ Non-gestational diabetes ${ }^{\mathrm{c}}$} \\
\hline No & 99.7 & 99.5 \\
\hline Yes & 0.3 & 0.5 \\
\hline
\end{tabular}

generations. The most common G2 outcome was obesity $(n=10,255 ; 12.8 \%)$; substantially fewer of the G2 women had any of the other outcomes: GDM $(n=431 ; 0.5 \%)$ and nongestational diabetes $(n=412 ; 0.5 \%)$.

G1 women with missing data on smoking during pregnancy were similar to those with complete data (electronic supplementary material [ESM] Table 1); among G2, those with missing smoking data were younger and more likely also to have missing data on BMI. There was no difference in prevalence of $\mathrm{G} 2$ obese women in the group with available G1 smoking data and missing G1 smoking data, respectively.

The accuracy of the smoking data for G1 women was evaluated by looking at the mean birthweight of the offspring of women in each G1 smoking category, yielding the following results: non-smoking mothers, $3,518 \mathrm{~g}$; mothers smoking 1-9 cigarettes/day, 3,320 g; and mothers smoking $>9$ cigarettes/day, 3,269 g .

Exposure to smoking in early pregnancy was associated with an increased risk of developing GDM in G2 women (adjusted OR [aOR] 1.62 [95\% CI 1.24, 2.13] for moderate exposure and 1.52 [95\% CI 1.12, 2.06] for heavy exposure) 
(Table 2). Exposed women were also more likely to be obese than non-exposed. A dose-response relationship was seen for obesity, where heavy smoking exposure was associated with a higher risk (aOR 1.58, 95\% CI 1.48, 1.68) than moderate exposure (aOR 1.36, 95\% CI 1.28, 1.44). With G1 smoking categories treated as continuous variables (coded as $0,1,2$ ), the $p$ value for trend (obesity) was $<0.01$.

For non-gestational diabetes, the results were not statistically significant in the moderately exposed group, but aORs were reduced in the heavily exposed group (0.66, 95\% CI 0.45, 0.96) (Table 2).

Results that were also adjusted for birthweight and gestational age resulted in attenuated ORs for GDM and strengthened ORs for obesity (Table 2).

The aORs for obesity were attenuated when the G2 women smoked themselves (Table 3). There was an interaction between G1 and G2 smoking in the obesity model $(p<0.001)$. Interaction terms were not significant in the other models (GDM, $p=0.81$; non-gestational diabetes, $p=0.57$; stratified results not shown).

Excluding G2 women with non-gestational diabetic mothers from the non-gestational diabetes model, or excluding G2 women with obese mothers from the obesity model did not change the OR for those outcomes (data not shown). Additionally adjusting the models for G1 BMI did not change the results (data not shown).
In the analysis that included only primiparous G2 women, compared with Table 2, the aORs for GDM were slightly increased for moderate exposure $(1.92,95 \%$ CI 1.41, 2.61), but remained basically unchanged in the heavily exposed group $(1.57,95 \%$ CI 1.10, 2.26). The results for obesity and non-gestational diabetes remained essentially the same (data not shown).

Including only women born in Sweden did not alter results (data not shown).

When only women delivering in Scania were included (where all pregnant women receive an OGTT), there was a slight strengthening of associations compared with the national sample (moderate smoking exposure aOR 2.01 [95\% CI 1.21, 3.34]; heavy smoking exposure aOR 2.05 [95\% CI 1.20, 3.50]).

\section{Discussion}

In these data, maternal smoking during early pregnancy was associated with an increased risk of daughters developing gestational diabetes and obesity in adulthood. For obesity a dose-response relationship was present. There was an inverse association between prenatal smoking exposure and non-gestational diabetes in the heavily exposed group. The associations remained after adjustment for age, parity, BMI, mode of delivery, gestational age and birthweight.

Table 2 OR $(95 \% \mathrm{CI})$ for the associations between maternal smoking during pregnancy (as categorised in the MBR) and daughters' risk of gestational diabetes, non-gestational diabetes and obesity (total $n=54,012$ )

\begin{tabular}{|c|c|c|c|c|c|c|}
\hline Outcome & Cases & Non-cases & $\begin{array}{l}\text { Unadjusted OR } \\
(95 \% \mathrm{CI})\end{array}$ & $\begin{array}{l}\text { Adjusted }^{\mathrm{a}} \\
\text { (without BMI) } \\
\text { OR }(95 \% \text { CI) }\end{array}$ & $\begin{array}{l}\text { Adjusted }^{\mathrm{b}} \text { (with BMI) } \\
\text { OR }(95 \% \mathrm{CI})\end{array}$ & $\begin{array}{l}\text { Adjusted for } \\
\text { birthweight and } \\
\text { gestational age }\end{array}$ \\
\hline \multicolumn{7}{|l|}{$\mathrm{GDM}^{\mathrm{d}}$} \\
\hline Non-smokers (ref) & 133 & 31,534 & 1 & 1 & 1 & 1 \\
\hline $1-9$ cigarettes/day ${ }^{\mathrm{e}}$ & 93 & 12,747 & $1.73(1.33,2.26)$ & $1.82(1.39,2.38)$ & $1.62(1.24,2.13)$ & $1.52(1.16,2.01)$ \\
\hline$>9$ cigarettes/day & 65 & 9,160 & $1.68(1.25,2.27)$ & $1.81(1.34,2.46)$ & $1.52(1.12,2.06)$ & $1.41(1.03,1.92)$ \\
\hline \multicolumn{7}{|l|}{ Non-gestational diabetes } \\
\hline Non-smokers (ref) & 172 & 31,667 & 1 & 1 & 1 & 1 \\
\hline 1-9 cigarettes/day & 75 & 12,840 & $1.08(0.82,1.41)$ & $1.11(0.84,1.46)$ & $1.07(0.82,1.42)$ & $1.09(0.82,1.44)$ \\
\hline$>9$ cigarettes/day & 33 & 9,225 & $0.66(0.45,0.96)$ & $0.69(0.47,1.01)$ & $0.66(0.45,0.96)$ & $0.66(0.45,0.97)$ \\
\hline \multicolumn{7}{|l|}{ Obesity (BMI >30) } \\
\hline Non-smokers (ref) & 3,674 & 28,165 & 1 & 1 & 1 & 1 \\
\hline 1-9 cigarettes/day & 1,990 & 10,925 & $1.40(1.32,1.48)$ & $1.36(1.28,1.44)$ & - & $1.45(1.36,1.54)$ \\
\hline$>9$ cigarettes/day & 1,636 & 7,622 & $1.65(1.54,1.75)$ & $1.58(1.48,1.68)$ & - & $1.71(1.60,1.83)$ \\
\hline
\end{tabular}

Only cases with complete data on all variables are included

${ }^{a}$ Models adjusted for woman's (G2) age, parity, mode of delivery and own smoking during early pregnancy

${ }^{\mathrm{b}}$ Obesity model not adjusted for BMI

${ }^{\mathrm{c}}$ Model adjusted for woman's (G2) age, parity, mode of delivery, own smoking during early pregnancy, BMI, birthweight and gestational age

${ }^{\mathrm{d}} \mathrm{G} 2$ women with non-gestational diabetes $(n=280)$ excluded from the GDM model

${ }^{\mathrm{e}} \mathrm{G} 1$ smoking during pregnancy as categorised in MBR

ref, reference 
Table 3 OR $(95 \%$ CI) for obesity by exposure to intrauterine tobacco smoking, stratified according to own smoking in G2

\begin{tabular}{|c|c|c|c|c|c|c|c|c|}
\hline \multirow[t]{2}{*}{ Outcome } & \multicolumn{4}{|c|}{ G2 Non-smokers $(n=45,124)$} & \multicolumn{4}{|c|}{ G2 Smokers ${ }^{\mathrm{a}}(n=8,888)$} \\
\hline & Cases & Non-cases & $\begin{array}{l}\text { Unadjusted } \\
\text { OR }(95 \% \mathrm{CI})\end{array}$ & $\begin{array}{l}\text { Adjusted } \mathrm{OR}^{\mathrm{b}} \\
(95 \% \mathrm{CI})\end{array}$ & Cases & Non-cases & $\begin{array}{l}\text { Unadjusted } \\
\text { OR }(95 \% \text { CI })\end{array}$ & $\begin{array}{l}\text { Adjusted OR }{ }^{\mathrm{b}} \\
(95 \% \mathrm{CI})\end{array}$ \\
\hline \multicolumn{9}{|l|}{ Obesity $(\mathrm{BMI}>30)$} \\
\hline Non-smokers (ref) & 3,209 & 25,457 & 1 & 1 & 465 & 2,708 & 1 & 1 \\
\hline $1-9$ cigarettes/day ${ }^{\mathrm{c}}$ & 1,484 & 8,461 & $1.39(1.30,1.49)$ & $1.38(1.29,1.47)$ & 506 & 2,464 & $1.20(1.04,1.37)$ & $1.19(1.04,1.37)$ \\
\hline$>9$ cigarettes/day & 1,158 & 5,355 & $1.72(1.59,1.85)$ & $1.70(1.58,1.83)$ & 478 & 2,267 & $1.23(1.07,1.41)$ & $1.23(1.07,1.41)$ \\
\hline
\end{tabular}

To our knowledge, only one previous study has investigated the association between in utero smoking exposure and subsequent GDM. Cupul-Uicab and colleagues found that exposed women were more likely to develop GDM than unexposed [6]. The present results confirm the findings on GDM in a large independent, population-based cohort, with the added advantage of a higher participation rate, as well as probably a more accurate exposure assessment, as women were asked about their current tobacco use, thus avoiding the estimation being based on recall of distant events. Further, at the time of data registration, the women were not aware of the study objectives, presumably minimising reporting bias. We were also able to evaluate whether a dose-response relation was present, which was not possible in the previous study. Whether the association is causal or due to unmeasured confounding remains unclear.

The interpretation of the results regarding non-gestational diabetes risk is more difficult because the distinction between type 1 and 2 diabetes cannot be reliably made with the MBR data. The majority of the cases of non-gestational diabetes in G2 were probably type 1; the prevalence of type 1 diabetes in the Swedish population is similar to the prevalence of diabetes in this cohort $(0.5 \%)$ [12]. An inverse association between smoking exposure and non-gestational diabetes in the group where G1 mothers were heavy smokers was observed. Under the assumption that the cases are type 1 diabetes, this is in accordance with previous studies [13-16]. Again, whether this association is due to a biological effect or confounding has yet to be ascertained.

A relation between pregnancy smoking and obesity in children has been reported consistently [3-5]. Regarding the continuance of the association in adulthood, however, results are conflicting $[6,7,17,18]$. For both the childhood and adult associations, some evidence suggests that it is due to residual confounding by unmeasured factors $[8,19,20]$, and the present data offer no opportunity to evaluate this possibility.

Nicotine has been linked to decreased appetite, as well as hyperphagia and weight gain upon cessation [21]. Among offspring of smoking mothers, this could provide a possible explanation for the reported phenomenon of increased postnatal weight gain and a higher rate of obesity [22]. Studies with rat models show that prenatal nicotine exposure is associated with increased adiposity and bodyweight, higher blood pressure and impaired glucose metabolism [9]. Changes in the hypothalamic regulation of appetite and satiety has been proposed as one underlying mechanism, as morphological changes have been described in these systems in offspring to rats malnourished during pregnancy [23]. Other reported effects of prenatal nicotine exposure include a higher rate of beta cell apoptosis and increased gene expression of transcription factors triggering adipocyte differentiation, which could be involved in the development of diabetes and obesity, respectively [24]. In addition, recent data show epigenetic changes in the offspring of smoking mothers [25].

Perhaps the main weakness of this study was our inability to take into account possible confounding due to educational level, income and other socioeconomic determinants of health. However, in the cohort used by Cupul-Uicab and colleagues to study the association between intrauterine smoking exposure and GDM risk, additional adjustment for education resulted in only a 5\% attenuation of the results (L. Cupul-Uicab, Center for Population Health Research, National Institute of Public Health, Cuernavaca, Morelos, Mexico, personal communication, 2012). While some error in reporting of smoking was likely, mean birthweight across G1 smoking categories shows the expected dose-response relationship, supporting the validity of the recorded data. GDM prevalence in the G2 generation in this cohort was $0.5 \%$, which is lower than the national prevalence reported elsewhere $(1.2-1.7 \%)$ [11, 26, 27]. However, this may possibly be explained by the cohort being younger, or by regional differences in screening methods for GDM. When only women giving birth in a region (Scania) where all pregnant women are offered an OGTT were included, the 
prevalence of GDM was in accordance with the numbers recorded above (1.2\%).

Another limitation is that the G2 generation is skewed towards the younger age spectrum, which is problematic mainly in two ways: (1) the prevalence of GDM and obesity is higher in older age groups, thus the G2 women might have been too young for latent risks to manifest, also yielding only a few cases with positive outcomes; (2) the results might not be generalisable to the whole population, and possibly even less so today when the average age that women in Sweden have their first child is close to 30 years [28].

A number of women had missing data on smoking behaviour that were not symmetrically distributed over the two generations (most missing in G1). Further, those with missing data on smoking in G2 were also more likely to have missing data on weight and height, in addition to being younger. However, the total number of missing cases in G2 was small $(n=4,071$ of a total of 100,175), and the prevalence of the outcomes was very similar. Prevalence of G2 obesity was not related to the availability of smoking data in G1.

Although short-term detrimental effects of smoking on the individual and her offspring are well known, such associations might extend into adulthood, making the incentive stronger for undertaking preventable measures, particularly as numbers in some countries point to an increase in daily smoking among young women [29].

\section{Conclusion}

In conclusion, these data show that women exposed to smoking during fetal life are at higher risk of developing gestational diabetes and obesity in adulthood. Possible residual confounding as an explanation for the findings cannot be excluded in this setting.

Funding The research was supported by Lund University and its Medical Faculty and the networks SIMSAM Early Life (with funding from The Swedish Research Council) and METALUND (with funding from Swedish Council for Working Life and Social Research). The research was also supported in part by the Intramural Research Program of the National Institutes of Health $(\mathrm{NIH})$, National Institute of Environmental Health Sciences (NIEHS).

Duality of interests The authors declare that there is no duality of interest associated with this manuscript.

Contribution statement MPL initiated the study. All authors contributed to the study design and interpretation of data. KM conducted the statistical analyses and drafted the manuscript. All authors critically revised the manuscript and approved the final, submitted version.

\section{References}

1. Cnattingius S (2004) The epidemiology of smoking during pregnancy: smoking prevalence, maternal characteristics, and pregnancy outcomes. Nicotine Tob Res 6:S125-S140

2. Aagaard-Tillery KM, Porter TF, Lane RH, Varner MW, Lacoursiere DY (2008) In utero tobacco exposure is associated with modified effects of maternal factors on fetal growth. Am J Obstet Gynecol 198:66.e61-e66

3. Ino T (2010) Maternal smoking during pregnancy and offspring obesity: meta-analysis. Pediatr Int 52:94-99

4. Oken E, Levitan EB, Gillman MW (2008) Maternal smoking during pregnancy and child overweight: systematic review and meta-analysis. Int J Obes (Lond) 32:201-210

5. Behl M, Rao D, Aagaard K et al (2013) Evaluation of the association between maternal smoking, childhood obesity, and metabolic disorders: a national toxicology program workshop review. Environ Health Perspect 121:170-180

6. Cupul-Uicab LA, Skjaerven R, Haug K, Melve KK, Engel SM, Longnecker MP (2012) In utero exposure to maternal tobacco smoke and subsequent obesity, hypertension, and gestational diabetes among women in the MoBa cohort. Environ Health Perspect 120:355-360

7. Power C, Jefferis BJ (2002) Fetal environment and subsequent obesity: a study of maternal smoking. Int J Epidemiol 31:413-419

8. Iliadou AN, Koupil I, Villamor E et al (2010) Familial factors confound the association between maternal smoking during pregnancy and young adult offspring overweight. Int $\mathrm{J}$ Epidemiol 39:1193-1202

9. Bruin JE, Gerstein HC, Holloway AC (2010) Long-term consequences of fetal and neonatal nicotine exposure: a critical review. Toxicol Sci 116:364-374

10. Socialstyrelsen (2003) The Swedish Medical Birth Register: a summary of content and quality. Socialstyrelsen, Stockholm

11. Persson M, Winkvist A, Mogren I (2007) No unified guidelines concerning gestational diabetes in Sweden. Noticeable differences between screening, diagnostics and management in maternal health services. Lakartidningen 104:3365-3369

12. Socialstyrelsen (2009) Folkhälsorapport 2009. Socialstyrelsen, Stockholm (in Swedish)

13. Svensson J, Carstensen B, Mortensen HB, Borch-Johnsen K (2005) Early childhood risk factors associated with type 1 diabetes: is gender important? Eur J Epidemiol 20:429-434

14. Lynch KF (2009) Perinatal determinants of type 1 diabetes: a social epidemiological perspective. Department of Clinical Sciences, Lund University, Malmö, Sweden

15. Marshall AL, Chetwynd A, Morris JA et al (2004) Type 1 diabetes mellitus in childhood: a matched case control study in Lancashire and Cumbria, UK. Diabet Med 21:1035-1040

16. Dahlquist G, Kallen B (1992) Maternal-child blood group incompatibility and other perinatal events increase the risk for early-onset type 1 (insulin-dependent) diabetes mellitus. Diabetologia 35:671-675

17. Power C, Atherton K, Thomas C (2010) Maternal smoking in pregnancy, adult adiposity and other risk factors for cardiovascular disease. Atherosclerosis 211:643-648

18. Thomas C, Hypponen E, Power C (2007) Prenatal exposures and glucose metabolism in adulthood: are effects mediated through birth weight and adiposity? Diabetes Care 30:918-924

19. Leary SD, Smith GD, Rogers IS, Reilly JJ, Wells JC, Ness AR (2006) Smoking during pregnancy and offspring fat and lean mass in childhood. Obesity (Silver Spring) 14:2284-2293

20. Howe LD, Matijasevich A, Tilling K et al (2012) Maternal smoking during pregnancy and offspring trajectories of height and adiposity: comparing maternal and paternal associations. Int J Epidemiol 41:722-732 
21. Williamson DF, Madans J, Anda RF, Kleinman JC, Giovino GA, Byers T (1991) Smoking cessation and severity of weight gain in a national cohort. N Engl J Med 324:739-745

22. Vik T, Jacobsen G, Vatten L, Bakketeig LS (1996) Pre- and postnatal growth in children of women who smoked in pregnancy. Early Hum Dev 45:245-255

23. Plagemann A, Harder T, Rake A, Melchior K, Rohde W, Dorner G (2000) Hypothalamic nuclei are malformed in weanling offspring of low protein malnourished rat dams. J Nutr 130:25822589

24. Somm E, Schwitzgebel VM, Vauthay DM, Aubert ML, Huppi PS (2009) Prenatal nicotine exposure and the programming of metabolic and cardiovascular disorders. Mol Cell Endocrinol 304:6977
25. Joubert BR, Haberg SE, Nilsen RM et al (2012) 450K epigenomewide scan identifies differential DNA methylation in newborns related to maternal smoking during pregnancy. Environ Health Perspect 120:1425-1431

26. Aberg A, Rydhstroem H, Frid A (2001) Impaired glucose tolerance associated with adverse pregnancy outcome: a population-based study in southern Sweden. Am J Obstet Gynecol 184:77-83

27. Ostlund I, Hanson U (2003) Occurrence of gestational diabetes mellitus and the value of different screening indicators for the oral glucose tolerance test. Acta Obstet Gynecol Scand 82:103-108

28. Statistics (2011) Sveriges befolkning 2011. Statistics Sweden, Stockholm (in Swedish)

29. Socialstyrelsen (2012) Folkhälsan i Sverige: Årsrapport 2012. Socialstyrelsen, Stockholm (in Swedish) 